\title{
Full Wave Modeling of Electromagnetic Scattering by an Object buried between two Rough Surfaces: Application to GPR
}

\author{
Marc Songolo, Nicolas Pinel, and Christophe Bourlier
}

\begin{abstract}
In this paper, we present an efficient numerical method to calculate the frequency and time responses of the field scattered by an object buried between two random rough surfaces. This method is called Generalized PILE (GPILE) method because it extends the PILE method which considers only two surfaces or an object buried under a surface. The GPILE method solves rigourously the Maxwell equations by using a simple matrix formulation. The obtained results have a straightforward physical interpretation and allow us to investigate the influence of the object buried between the two rough surfaces. We distinguish the primary echo of the upper surface, the multiple echoes coming from the lower surface and those arising from the object. The GPILE method is applied to simulate the Ground Penetrating Radar (GPR) signal at nadir. The resulting time response helps the user to detect the presence of the object buried between the two random rough surfaces.
\end{abstract}

Index Terms-Electromagnetic Scattering, Rough Surfaces, Method of Moments, Propagation Inside Layer Expansion, Ground Penetrating Radar.

\section{INTRODUCTION}

$\mathbf{E}$ LECTROMAGNETIC scattering from an object buried under a rough surface has attracted much interest for several decades. In order to solve this problem, some asymptotic and rigorous models have been developped. The asymptotic (or approximate) models are based on simplifying assumptions. Rigorous models are less restrictive and thus find a large field of validity. Numerical methods for rigorous models can be classified into two families: differential equations methods, where discretization uses Finite Element Method (FEM) [1], [2] or Finite Difference Time Domain (FDTD) method [3], [4] and the integral boundary methods, where the discretization uses the Method of Moments (MoM) [5], [6]. The MoM offers several advantages over FDTD. Indeed, the MoM discretizes the boundaries (here the interfaces), while the FDTD discretizes the space. For rough interfaces, the mesh grid must be refined in the neighbourhood of the interfaces for the FDTD in order to follow the surface profiles, which increases the memory space requirements, unlike for the MoM. The main

Marc Songolo is with Icam Ouest School of Engineering - Nantes campus, Carquefou, France, with IETR, Polytech Nantes, University of Nantes, Nantes, France, and University of Lubumbashi, Lubumbashi, Republic Democratic of the Congo e-mail: (see marc.songolo@gmail.com).

Nicolas Pinel is with Icam Ouest School of Engineering - Nantes campus, Carquefou, France, with IETR, Polytech Nantes, University of Nantes, Nantes, France,

Christophe Bourlier is with IETR, Polytech Nantes, University of Nantes, Nantes, France.

Manuscript received July 06, 2021; revised August 30, 2021 drawback of MoM is that it can be applied only for interfaces separated by homogenous media and for a single frequency. Thus, the calculation of a time response necessitate to apply MoM $N_{f}$ times, where $N_{f}$ is the number of frequencies.

In the case of a single rough surface, fast methods for solving the linear system obtained by the MoM have been developed in order to reduce the number of operations as well as the storage memory space. For example, the Method of Ordered Multiple Interactions (MOMI) [7]. The major drawback of this method is its slower convergence for the dielectric case. The Forward-Backward (FB) method [8] adapts better to dielectric interfaces [9]. An accelerated version of the FB uses the spectral representation of Green's function (FB-SA) [10]. The Banded-Matrix-Iterative-Approach (BMIA) method [11], [12] aims to accelerate the matrix-vector products. An improved version of this method gave rise to the Banded-MatrixIterative-Approach/CAnonical Grid (BMIA-CAG) [13].

In the case of scattering by two rough surfaces, some methods have been devoted to obtaining a rigorous solution: the Extended Boundary Condition Method (EBCM) [14], the Forward-Backward with Spectral Acceleration (FBSA) method [15], the Steepest Descent Fast Multipode Method (SDFMM) [16]. However, all these methods have some constraints. In contrast, the Propagation Inside-Layed Expansion (PILE) method [17] is rigorous, of a simple mathematical formulation and has an intuitive physical interpretation. This method has been accelerated by using the fast algorithm BMIA/CAG to give the PILE-BMIA/CAG method [18]. Another acceleration of the PILE method adapts the spectral acceleration (SA), both to local interactions and to the coupling steps and gives the PILE-FBSA method [19]. The PILE method has been adapted to scattering from an object buried under a rough surface [20]. In the same spirit, the PILE method has been extended to the more general case of the scattering from two illuminated scatterers and gives Extended PILE method [21]. A generalization of the PILE method (GPILE: Generalized Propagation Inside-Layed Expansion) has been presented in [22], to calculate the electromagnetic field scattered by three superimposed surfaces.

In this paper, we consider an object buried inside a layer with two rough interfaces and we assume that all media are homogeneous. We construct a rigorous model for this problem from an integral boundary method in the frequency domain, where the discretization uses the MoM. To reduce the complexity of the inversion of the impedance matrix obtained by the MoM, we extend the PILE algorithm [17] to the case 
of three scatterers by forming a composite scatterer made up of the object and the lower surface. The purpose of this paper is to present detailed numerical studies of the GPILE method for an object buried between two rough surfaces. The results are presented both in the frequency domain to highlight certain elements such as the energy distribution of the signal in a frequency band, and in the time domain to highlight the variations in the amplitude of the signal over a given period of time. This will then allow us to distinguish the primary echo from the upper surface and the multiple echoes from the object and/or the lower surface. The study is expected to give a better understanding of the propagation phenomena in complex media such as that composed of an object buried between two rough surfaces.

In what follows, we expose the geometry of the problem in section II. Section III presents the GPILE method based on the PILE method. Then, we establish detailed calculations from this method. Section IV presents some numerical simulations for a single frequency. Section $\mathrm{V}$ provides simulations for Ground Penetrating Radar (GPR) signals for a realistic scenario for the problem under study.

\section{GEOMETRY OF THE PROBLEM}

Consider in Fig. 1 two random rough surfaces $S_{1}$ and $S_{3}$ invariant along the $\boldsymbol{y}$ axis and an object delimited by the surface $S_{2}$ buried between the interfaces $S_{1}$ and $S_{3}$. The two surface heights are generated by a Gaussian probability density function (PDF) and a Gaussian autocorrelation function (ACF), and we assume that they do not intercept each other or with the object. The three interfaces separate four homogeneous media: the upper medium, $\Omega_{0}$, assumed to be vaccum, the intermediate medium, $\Omega_{1}$, which constitutes a layer, the buried object which constitutes the medium $\Omega_{2}$, and the lower medium, $\Omega_{3}$, which we consider to be dielectric. Consider an incident wave $\psi_{\text {inc }}(\boldsymbol{r})$ in the plane $(\hat{\boldsymbol{x}}, \hat{\boldsymbol{z}})$, at an incidence angle $\theta_{\text {inc }}$ defined relatively to the axis $\boldsymbol{z}$ counterclockwise, as shown in Fig.1.

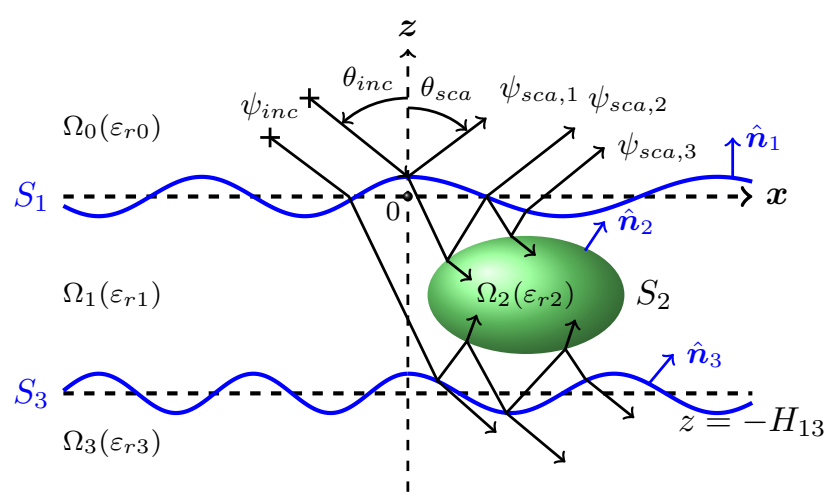

Fig. 1. Electromagnetic scattering from an object buried between two 1-D rough surfaces (2-D problem: plane $(\hat{\boldsymbol{x}}, \hat{\boldsymbol{z}}))$.

Since surfaces have a finite length, edge diffraction occurs because the incident field does not vanish at the edges of the surfaces. To reduce this phenomenon in the study of scattering from rough surfaces, a tapered incident wave must be used instead of a plane incident wave. Here, we will apply the Thorsos wave, as described in [23], with an attenuation parameter $g$ which controls the extent of the incident wave.

\section{Method of Moments}

\section{A. Impedance Matrix}

The integral equation method consist in evaluating the currents $\psi_{i}$ and their normal derivatives $\partial \psi_{i} / \partial n_{i}$ on each surface $S_{i}(i=\{1,2,3\})$ and in deducing the scattered fields in each medium by using Huygens' principle. From the MoM, the boundary integral equations are discretized on each surface of the scatterer, leading to the linear system $\bar{Z} \boldsymbol{X}=\boldsymbol{b}$, where the impedance matrix is

$$
\overline{\boldsymbol{Z}}=\left(\begin{array}{lll}
\overline{\boldsymbol{Z}}_{11} & \overline{\boldsymbol{Z}}_{21} & \overline{\boldsymbol{Z}}_{31} \\
\overline{\boldsymbol{Z}}_{12} & \overline{\boldsymbol{Z}}_{22} & \overline{\boldsymbol{Z}}_{32} \\
\overline{\boldsymbol{Z}}_{13} & \overline{\boldsymbol{Z}}_{23} & \overline{\boldsymbol{Z}}_{33}
\end{array}\right)
$$

The impedance matrix is of size $2\left(\sum_{i=1}^{3} N_{i}\right) \times 2\left(\sum_{i=1}^{3} N_{i}\right)$, where $N_{i}$ is the number of samples on $S_{i}$. It is valuable to notice that all the coupling matrices are non-zero, since all the interfaces have direct interaction between them (unlike the case of three interfaces treated in [22]). The unknown vectors $\boldsymbol{X}_{i}$ containing the surface currents and their normal derivatives are written as

$$
\boldsymbol{X}_{i}=\left[\psi_{i}\left(\boldsymbol{r}_{1}\right) \cdots \psi_{i}\left(\boldsymbol{r}_{N_{i}}\right) \quad \frac{\partial \psi_{i}\left(\boldsymbol{r}_{1}\right)}{\partial n_{i}} \cdots \frac{\partial \psi_{i}\left(\boldsymbol{r}_{N_{i}}\right)}{\partial n_{i}}\right]^{T}
$$

where $\boldsymbol{r}_{p \in\left\{1 ; N_{i}\right\}} \in S_{i}, i=\{1,3\}$ for the two surfaces, and $\boldsymbol{r}_{p \in\left\{1 ; N_{2}\right\}} \in S_{2}$ for the object.

The term $\boldsymbol{b}$ is the source and contains information on the incident field:

$$
\boldsymbol{b}=\left[\begin{array}{lll}
\boldsymbol{b}_{1} & \boldsymbol{b}_{2} & \boldsymbol{b}_{3}
\end{array}\right]^{T} .
$$

In the absence of one of the scatterers, one can find the impedance matrix conform to the case of the other two scatterers. This remark establishes a theoretical and rapid validation of the MoM compared to existing work on electromagnetic scattering, in the case of two rough surfaces [17], an object below a rough surface [20], an object above a rough surface [21].

\section{B. Perfect Conductor Cases}

We can consider the cases where the object and/or the lower surface are perfect conductors. In these cases, the impedance matrices are simplified. If the object is a perfect conductor, then $\psi_{2}$ vanishes on the interface of the object in Transverse Electric polarization (TE, Dirichlet boundary conditions) and the only unknown is $\partial \psi_{2} / \partial n_{2}$. On the other hand, in Transverse Magnetic polarization (TM, Neumann boundary conditions), $\partial \psi_{2} / \partial n_{2}$ vanishes on the interface of the object and the only unknown is $\psi_{2}$. So we have

$$
\left\{\begin{aligned}
\mathrm{TE}: \overline{\boldsymbol{Z}}_{22} & =\overline{\boldsymbol{B}}_{2}, \overline{\boldsymbol{Z}}_{12}=\left[\begin{array}{ll}
\overline{\boldsymbol{A}}_{12} & \rho_{10} \overline{\boldsymbol{B}}_{12}
\end{array}\right], \\
\overline{\boldsymbol{Z}}_{32} & =\left[\begin{array}{ll}
\overline{\boldsymbol{A}}_{32} & \overline{\boldsymbol{B}}_{32}
\end{array}\right], \overline{\boldsymbol{Z}}_{21}=\left[\begin{array}{ll}
\overline{\mathbf{0}} & \overline{\boldsymbol{B}}_{21}
\end{array}\right]^{T}, \\
\overline{\boldsymbol{Z}}_{23} & =\left[\begin{array}{ll}
\overline{\boldsymbol{B}}_{23} & \overline{\mathbf{0}}
\end{array}\right]^{T} . \\
\mathrm{TM}: \overline{\boldsymbol{Z}}_{22} & =\overline{\boldsymbol{A}}_{2}, \overline{\boldsymbol{Z}}_{12}=\left[\begin{array}{ll}
\overline{\boldsymbol{A}}_{12} & \rho_{10} \overline{\boldsymbol{B}}_{12}
\end{array}\right], \\
\overline{\boldsymbol{Z}}_{32} & =\left[\begin{array}{ll}
\overline{\boldsymbol{A}}_{32} & \overline{\boldsymbol{B}}_{32}
\end{array}\right], \overline{\boldsymbol{Z}}_{21}=\left[\begin{array}{ll}
\overline{\mathbf{0}} & \overline{\boldsymbol{A}}_{21}
\end{array}\right]^{T}, \\
\overline{\boldsymbol{Z}}_{23} & =\left[\begin{array}{ll}
\overline{\boldsymbol{A}}_{23} & \overline{\mathbf{0}}
\end{array}\right]^{T} .
\end{aligned}\right.
$$


where $\rho_{10}=\epsilon_{r 1} / \epsilon_{r 0}$ with $\epsilon_{r 0}$ and $\epsilon_{r 1}$ the relative permitivities of $\Omega_{0}$ and $\Omega_{1}$, respectively.

On the other hand, if the lower surface is a perfect conductor, then

$$
\left\{\begin{aligned}
\mathrm{TE}: \overline{\boldsymbol{Z}}_{33} & =\overline{\boldsymbol{B}}_{3}, \overline{\boldsymbol{Z}}_{13}=\left[\begin{array}{ll}
\overline{\boldsymbol{A}}_{13} & \rho_{10} \overline{\boldsymbol{B}}_{13}
\end{array}\right], \\
\overline{\boldsymbol{Z}}_{23} & =\left[\begin{array}{ll}
\overline{\boldsymbol{A}}_{23} & \overline{\boldsymbol{B}}_{23}
\end{array}\right], \overline{\boldsymbol{Z}}_{31}=\left[\begin{array}{ll}
\overline{\mathbf{0}} & \overline{\boldsymbol{B}}_{31}
\end{array}\right]^{T}, \\
\overline{\boldsymbol{Z}}_{32} & =\left[\begin{array}{ll}
\overline{\boldsymbol{B}}_{32} & \overline{\mathbf{0}}
\end{array}\right]^{T} . \\
\mathrm{TM}: \overline{\boldsymbol{Z}}_{33} & =\overline{\boldsymbol{A}}_{3}, \overline{\boldsymbol{Z}}_{13}=\left[\begin{array}{ll}
\overline{\boldsymbol{A}}_{13} & \rho_{10} \overline{\boldsymbol{B}}_{13}
\end{array}\right] \\
\overline{\boldsymbol{Z}}_{23} & =\left[\begin{array}{ll}
\overline{\boldsymbol{A}}_{23} & \overline{\boldsymbol{B}}_{23}
\end{array}\right], \overline{\boldsymbol{Z}}_{31}=\left[\begin{array}{ll}
\overline{\mathbf{0}} & \overline{\boldsymbol{A}}_{31}
\end{array}\right]^{T}, \\
\overline{\boldsymbol{Z}}_{32} & =\left[\begin{array}{ll}
\overline{\boldsymbol{A}}_{32} & \overline{\mathbf{0}}
\end{array}\right]^{T} .
\end{aligned}\right.
$$

From these two cases, we can find the case where both the object and the lower surface are perfect conductors.

\section{GPILE}

To reduce the complexity of the inversion of the impedance matrix obtained by the MoM, we extend the PILE algorithm [17] to the case of three scatterers by considering on the one hand the upper surface as scatterer 1 , and on the other hand the object and the lower surface as a single scatterer, which we call composite scatterer 2 . Let us rewrite impedance matrices for this structure as:

$$
\begin{aligned}
& \overline{\boldsymbol{P}}_{11}=\overline{\boldsymbol{Z}}_{11}, \quad \overline{\boldsymbol{P}}_{22}=\left(\begin{array}{ll}
\overline{\boldsymbol{Z}}_{22} & \overline{\boldsymbol{Z}}_{32} \\
\overline{\boldsymbol{Z}}_{23} & \overline{\boldsymbol{Z}}_{33}
\end{array}\right), \\
& \overline{\boldsymbol{P}}_{12}=\left(\begin{array}{lll}
\overline{\boldsymbol{Z}}_{12} & \overline{\boldsymbol{Z}}_{13}
\end{array}\right)^{T}, \quad \overline{\boldsymbol{P}}_{21}=\left(\begin{array}{ll}
\overline{\boldsymbol{Z}}_{21} & \overline{\boldsymbol{Z}}_{31}
\end{array}\right) .
\end{aligned}
$$

The PILE method in the case of two scatterers consists in inverting the impedance matrix

$$
\overline{\boldsymbol{Z}}=\left(\begin{array}{ll}
\overline{\boldsymbol{P}}_{11} & \overline{\boldsymbol{P}}_{21} \\
\overline{\boldsymbol{P}}_{12} & \overline{\boldsymbol{P}}_{22}
\end{array}\right)
$$

by decomposition of domains from the Taylor series expansion of the inverse of the Schur complement [17]:

$$
\bar{Z}^{-1}=\left(\begin{array}{cc}
\bar{T} & \bar{U} \\
\bar{V} & \bar{W}
\end{array}\right)
$$

where the matrices $\overline{\boldsymbol{T}}, \overline{\boldsymbol{U}}, \overline{\boldsymbol{V}}$ and $\overline{\boldsymbol{W}}$ are expressed as functions of four block matrices of $\bar{Z}$ as follows

$$
\left\{\begin{aligned}
\overline{\boldsymbol{T}} & =\left[\overline{\boldsymbol{P}}_{11}-\overline{\boldsymbol{P}}_{21} \overline{\boldsymbol{P}}_{22}^{-1} \overline{\boldsymbol{P}}_{12}\right]^{-1} \\
\overline{\boldsymbol{U}} & =-\overline{\boldsymbol{T}} \overline{\boldsymbol{P}}_{21} \overline{\boldsymbol{P}}_{22}^{-1} \\
\overline{\boldsymbol{V}} & =-\overline{\boldsymbol{P}}_{22}^{-1} \overline{\boldsymbol{P}}_{12} \overline{\boldsymbol{T}} \\
\overline{\boldsymbol{W}} & =\overline{\boldsymbol{P}}_{22}^{-1}+\overline{\boldsymbol{P}}_{22}^{-1} \overline{\boldsymbol{P}}_{12} \overline{\boldsymbol{T}}_{21} \overline{\boldsymbol{P}}_{22}^{-1}
\end{aligned}\right.
$$

The PILE method then makes it possible to obtain the surface currents $\boldsymbol{Y}_{1}$ and $\boldsymbol{Y}_{2}$ on the two scatterers

$$
\left\{\begin{array}{l}
\boldsymbol{Y}_{1}=\left(\sum_{p=0}^{P} \overline{\boldsymbol{N}}_{c}^{p}\right) \overline{\boldsymbol{P}}_{11}^{-1} \boldsymbol{b}_{1} \\
\boldsymbol{Y}_{2}=-\overline{\boldsymbol{P}}_{22}^{-1} \overline{\boldsymbol{P}}_{12} \boldsymbol{Y}_{1}
\end{array}\right.
$$

where

$$
\overline{\boldsymbol{N}}_{c}=\overline{\boldsymbol{P}}_{11}^{-1} \overline{\boldsymbol{P}}_{21} \overline{\boldsymbol{P}}_{22}^{-1} \overline{\boldsymbol{P}}_{12}
$$

is the coupling matrix of the composite scatterer 2 towards the scatterer 1 and thus brings the contributions of the composite scatterer 2 to the scatterer 1 .
The convergence of this method is based on the value of the spectral norm of $\overline{\boldsymbol{N}}_{c}$. Denoted $\left\|\overline{\boldsymbol{N}}_{c}\right\|_{\mathrm{sr}}$, this norm is always strictly smaller than 1, which ensures the convergence of the algorithm.

We now want to detail the calculations of the currents on each surface in the spirit of the original PILE method. To do this, we denote the surface currents $\boldsymbol{X}_{1}, \boldsymbol{X}_{2}$ and $\boldsymbol{X}_{3}$ respectively. Note that $\boldsymbol{X}_{1}=\boldsymbol{Y}_{1}$, because scatterer 1 corresponds to the upper surface. It is then sufficient to detail the calculations of the characteristic matrix $\overline{\boldsymbol{N}}_{c}$ to obtain an expression equivalent to $\boldsymbol{X}_{1}$, the only issue being to invert the matrix $\overline{\boldsymbol{P}}_{22}$. By reusing the inverse of the Schur complement algorithm, we obtain

$$
\begin{aligned}
\overline{\boldsymbol{N}}_{c} & =\overline{\boldsymbol{Z}}_{11}^{-1} \overline{\boldsymbol{Z}}_{21}\left(\overline{\boldsymbol{T}} \overline{\boldsymbol{Z}}_{12}+\overline{\boldsymbol{U}} \overline{\boldsymbol{Z}}_{13}\right) \\
& +\overline{\boldsymbol{Z}}_{11}^{-1} \overline{\boldsymbol{Z}}_{31}\left(\overline{\boldsymbol{V}} \overline{\boldsymbol{Z}}_{12}+\overline{\boldsymbol{W}} \overline{\boldsymbol{Z}}_{13}\right)
\end{aligned}
$$

where the matrices $\overline{\boldsymbol{T}}, \overline{\boldsymbol{U}}, \overline{\boldsymbol{V}}$ and $\overline{\boldsymbol{W}}$ are given by (9) as functions of four blocks of the matrix $\overline{\boldsymbol{P}}_{22}$.

Recall in addition that, as the matrix $\overline{\mathbf{N}}_{c}$ is the sum of the contributions of the object and of the lower surface on the upper surface, we can explicitly highlight the two contributions by noting:

$$
\overline{\boldsymbol{N}}_{c, 21}=\overline{\boldsymbol{Z}}_{11}^{-1} \overline{\boldsymbol{Z}}_{21}\left(\overline{\boldsymbol{T}} \overline{\boldsymbol{Z}}_{12}+\overline{\boldsymbol{U}}_{\left.\overline{\boldsymbol{Z}}_{13}\right)}\right.
$$

and

$$
\overline{\boldsymbol{N}}_{c, 31}=\overline{\boldsymbol{Z}}_{11}^{-1} \overline{\boldsymbol{Z}}_{31}\left(\overline{\boldsymbol{V}} \overline{\boldsymbol{Z}}_{12}+\overline{\boldsymbol{W}} \overline{\boldsymbol{Z}}_{13}\right) .
$$

In reality, the matrices $\overline{\boldsymbol{N}}_{c, 21}$ and $\overline{\boldsymbol{N}}_{c, 31}$ are characteristic matrices for the principal interactions (those coming from the upper surface towards the object or the lower surface) and bring back the contributions of the object and the lower surface towards the upper surface, respectively.

By injecting relations (9) into the characteristic matrices (13) and (14), we obtain their approximations by the Taylor series expansion (with truncation to the order $Q$ )

$$
\begin{aligned}
\overline{\boldsymbol{N}}_{c, 21} \approx \sum_{q=0}^{Q} \overline{\boldsymbol{M}}_{c, 21}^{q}= & \overline{\boldsymbol{Z}}_{11}^{-1} \overline{\boldsymbol{Z}}_{21}\left(\sum_{q=0}^{Q} \overline{\boldsymbol{M}}_{c, 32}^{q}\right) \times \\
& \overline{\boldsymbol{Z}}_{22}^{-1}\left(\overline{\boldsymbol{Z}}_{12}-\overline{\boldsymbol{Z}}_{12}^{\prime}\right)
\end{aligned}
$$

and

$$
\begin{aligned}
& \overline{\boldsymbol{N}}_{c, 31} \approx \sum_{q=0}^{Q} \overline{\boldsymbol{M}}_{c, 31}^{q}=\overline{\boldsymbol{Z}}_{11}^{-1} \overline{\boldsymbol{Z}}_{31}\left(\overline{\boldsymbol{Z}}_{33}\right)^{-1} \times \\
& {\left[\overline{\boldsymbol{Z}}_{13}-\overline{\boldsymbol{Z}}_{23}\left(\sum_{q=0}^{Q} \overline{\boldsymbol{M}}_{c, 32}^{q}\right) \overline{\boldsymbol{Z}}_{22}^{-1}\left(\overline{\boldsymbol{Z}}_{12}-\overline{\boldsymbol{Z}}_{12}^{\prime}\right)\right]}
\end{aligned}
$$

where

$$
\overline{\mathbf{N}}_{c, 32}=\overline{\boldsymbol{Z}}_{22}^{-1} \overline{\boldsymbol{Z}}_{32} \overline{\boldsymbol{Z}}_{33}^{-1} \overline{\boldsymbol{Z}}_{23}
$$

is the characteristic matrix which brings back the contributions from the lower surface to the object, taking into account a secondary interaction at the order $q$. The matrix $\bar{Z}_{12}^{\prime}=$ $\bar{Z}_{32} \bar{Z}_{33}^{-1} \bar{Z}_{13}$ represents the coupling matrix between the upper surface and the object via the lower surface. 
We present in Appendix B the calculations of surface currents by the substitution method. An equivalent expression to the characteristic matrix (16) obtained by this method is

$$
\begin{aligned}
\overline{\boldsymbol{N}}_{c, 31} \approx \sum_{q=0}^{Q} \overline{\boldsymbol{M}}_{c, 31}^{q}= & \overline{\boldsymbol{Z}}_{11}^{-1} \overline{\boldsymbol{Z}}_{31}\left(\sum_{q=0}^{Q} \overline{\boldsymbol{M}}_{c, 23}^{q}\right) \times \\
& \overline{\boldsymbol{Z}}_{33}^{-1}\left(\overline{\boldsymbol{Z}}_{13}-\overline{\boldsymbol{Z}}_{13}^{\prime}\right)
\end{aligned}
$$

where

$$
\overline{\boldsymbol{M}}_{c, 23}=\overline{\boldsymbol{Z}}_{33}^{-1} \overline{\boldsymbol{Z}}_{23} \overline{\boldsymbol{Z}}_{22}^{-1} \overline{\boldsymbol{Z}}_{32},
$$

and the matrix $\bar{Z}_{13}^{\prime}=\bar{Z}_{23} \bar{Z}_{22}^{-1} \bar{Z}_{12}$ represents the coupling matrix between the upper surface and the lower surface via the object.

Expression (18) brings out the symmetry of the problem instead of (16). It will be used for the rest of the paper. Moreover, the contribution matrix of the object and the lower surface each consists of two contributions, one of which being direct and the other being indirect. It is the coupling matrices $\bar{Z}_{12}$ and $\overline{\boldsymbol{Z}}_{12}^{\prime}$ which make it possible to quantify these contributions for the object. The coupling matrices $\bar{Z}_{13}$ and $\bar{Z}_{13}^{\prime}$ play the same role for the lower surface.

By regrouping in one expression the two contributions to calculate the total currents on the upper surface, we obtain:

$$
\boldsymbol{X}_{1}=\sum_{p=0}^{P}\left(\sum_{q=0}^{Q} \overline{\boldsymbol{M}}_{c, 21}^{q}+\sum_{q=0}^{Q} \overline{\boldsymbol{M}}_{c, 31}^{q}\right)^{p} \overline{\boldsymbol{Z}}_{11}^{-1} \boldsymbol{b}_{1} .
$$

Finaly,

$$
\boldsymbol{X}_{1}=\sum_{p=0}^{P}\left(\sum_{q=0}^{Q} \overline{\boldsymbol{M}}_{c}^{q}\right)^{p} \overline{\boldsymbol{Z}}_{11}^{-1} \boldsymbol{b}_{1} .
$$

It is important to note that the orders $P$ and $Q$ are arbitrary. In fact, according to the construction of the method (see expressions (10)), the main order $p$ represents the round trips of the wave between the upper surface and the object or the lower surface. As for the secondary order $q$, it represents the round trips of the wave between the object and the lower surface, and vice versa.

The calculations of the currents on the other interfaces are done by the same types of algebraic manipulations, and we obtain for the object

$$
\boldsymbol{X}_{2}=-\left(\overline{\boldsymbol{T}} \overline{\boldsymbol{Z}}_{12}+\overline{\boldsymbol{U}} \overline{\boldsymbol{Z}}_{13}\right) \boldsymbol{X}_{1}=-\overline{\boldsymbol{T}}\left(\overline{\boldsymbol{Z}}_{12}-\overline{\boldsymbol{Z}}_{12}^{\prime}\right) \boldsymbol{X}_{1}
$$

from where

$$
\boldsymbol{X}_{2}=-\left(\sum_{q=0}^{Q} \overline{\boldsymbol{M}}_{c, 32}^{q}\right) \overline{\boldsymbol{Z}}_{22}^{-1}\left(\overline{\boldsymbol{Z}}_{12}-\overline{\boldsymbol{Z}}_{12}^{\prime}\right) \boldsymbol{X}_{1}
$$

and for the lower surface

$$
\begin{aligned}
\boldsymbol{X}_{3} & =-\left(\overline{\boldsymbol{V}} \overline{\boldsymbol{Z}}_{12}+\overline{\boldsymbol{W}} \overline{\boldsymbol{Z}}_{13}\right) \boldsymbol{X}_{1} \\
& =-\overline{\boldsymbol{V}}\left(\overline{\boldsymbol{Z}}_{12}-\overline{\boldsymbol{Z}}_{12}^{\prime}\right) \boldsymbol{X}_{1}-\overline{\boldsymbol{Z}}_{33}^{-1} \overline{\boldsymbol{Z}}_{13} \boldsymbol{X}_{1}
\end{aligned}
$$

from where

$$
\begin{aligned}
\boldsymbol{X}_{3} & =\overline{\boldsymbol{Z}}_{33}^{-1} \overline{\boldsymbol{Z}}_{23}\left(\sum_{q=0}^{Q} \overline{\boldsymbol{M}}_{c, 32}^{q}\right) \overline{\boldsymbol{Z}}_{22}^{-1}\left(\overline{\boldsymbol{Z}}_{12}-\overline{\boldsymbol{Z}}_{12}^{\prime}\right) \boldsymbol{X}_{1} \\
& -\overline{\boldsymbol{Z}}_{33}^{-1} \overline{\boldsymbol{Z}}_{13} \boldsymbol{X}_{1} .
\end{aligned}
$$

The calculations of the currents on the lower surface by applying the substitution method (see Appendix B) make it possible to obtain

$$
\boldsymbol{X}_{3}=-\left(\sum_{q=0}^{Q} \overline{\boldsymbol{M}}_{c, 23}^{q}\right) \overline{\boldsymbol{Z}}_{33}^{-1}\left(\overline{\boldsymbol{Z}}_{13}-\overline{\boldsymbol{Z}}_{13}^{\prime}\right) \boldsymbol{X}_{1} .
$$

The expressions of the currents on the object and on the lower surface have a physical interpretation in accordance with the EPILE method [21].

Remark 1: We can calculate the currents on the upper surface according to the expression (21) and calculate them on the object and the lower surface by using the EPILE algorithm [21]. By considering that the object and the lower surface are illuminated by the fields $-\overline{\boldsymbol{Z}}_{12} \boldsymbol{X}_{1}$ and $-\overline{\boldsymbol{Z}}_{13} \boldsymbol{X}_{1}$ respectively, we obtain relations (23) and (26). The sign (-) in the fields comes from the fact that the two scatterers are in the transmitted medium with respect to the upper surface.

Remark 2: The fundamental difference between (10) and (21) is the fact that in (10), $Q=\infty$, because the impedance matrix $\overline{\boldsymbol{P}}_{22}$ (for the lower surface and the object) is inverted directly, while in (21), $Q=0,1,2$, etc. In this way $\overline{\boldsymbol{N}}_{c}=\sum_{q=0}^{Q} \overline{\boldsymbol{M}}_{c}^{q}$, and the expression (21) allows us to study numerically the contributions of the object and of the lower surface for each scattering.

Remark 3: It is valuable to note that if instead of an object we had a surface, there would not be direct interaction between the upper surface and the lower surface, which would result in $\overline{\boldsymbol{Z}}_{13}=\overline{\mathbf{0}}$ (and therefore $\overline{\boldsymbol{Z}}_{31}=\overline{\mathbf{0}}$ ). It would follow that $\overline{\boldsymbol{N}}_{c, 31}=\overline{\mathbf{0}}$ and one would find a GPILE method equivalent to that developed in [22] for the case of three superimposed interfaces.

For the numerical simulations, we implement expression (21) to calculate the currents on the upper surface and expressions (23) and (26) for the calculation of the currents on the object and on the lower surface, respectively. The characteristic matrices will be approximated by expressions (15) and (18). To avoid calculating products of matrices when approximating the characteristic matrices, we will start the process by calculating the currents and their normal derivatives on the upper surface for $P=0$, which amounts to calculating a matrix-vector product. Having the current vector on the upper surface at this order, we can then calculate it for the order $P=1$ by matrix-vector products and so on. This procedure is well suited for calculating currents for any value of $Q$. In this way, the currents on the object and on the lower surface will also be calculated for any value $P$ and $Q$ by matrix-vector products.

\section{NUMERICAL RESULTS FOR A SINGLE FREQUENCY}

The GPILE method was derived from the PILE method by coupling the upper surface, and the object plus the lower surface as a single composite scatterer. Thus, by decoupling the object and the lower surface, we can numerically study the impact of the orders $p$ and $q$ on the convergence of the algorithm, as well as the contributions of the object and of the lower surface on the scattered field in the incidence medium. In addition, the orders $p$ and $q$ make it possible to analyse 


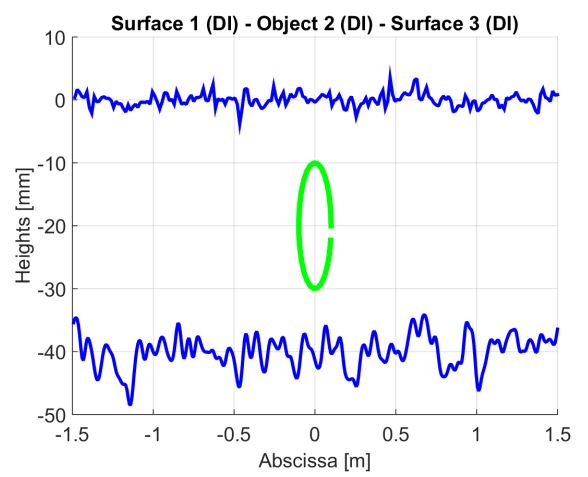

Fig. 2. Surface heights versus their abscissa for Gaussian PDF and ACF, with $\sigma_{\mathrm{h}, 1}=1 \mathrm{~mm}$ and $\sigma_{\mathrm{h}, 3}=2.5 \mathrm{~mm}, L_{\mathrm{ch}, 1}=15 \mathrm{~mm}$ and $L_{\mathrm{ch}, 3}=30 \mathrm{~mm}$. The center of the cylinder is $C=(0,-0.5 H)$, and $a=100 \mathrm{~mm}$ and $b=10 \mathrm{~mm}$.

the influence of the primary echo of the upper surface and the multiple echoes in the intermediate medium.

Consider an elliptical cylinder of semi-major axis $a=100$ $\mathrm{mm}$ and semi-minor axis $b=10 \mathrm{~mm}$ buried between two rough surfaces of length $3000 \mathrm{~mm}$, which are generated by a Gaussian probability density function (PDF) and a Gaussian autocorrelation function (ACF). Moreover, we assume that these surfaces are not correlated. The three interfaces separate four homogeneous media $\left(\Omega_{0}, \Omega_{1}, \Omega_{2}, \Omega_{3}\right)$ as described in section II. The standard deviations of the heights of the two random rough surfaces are $\sigma_{\mathrm{h}, 1}=1 \mathrm{~mm}$ and $\sigma_{\mathrm{h}, 3}=2.5$ $\mathrm{mm}$, respectively. Their correlation lengths are $L_{\mathrm{ch}, 1}=15$ $\mathrm{mm}$ and $L_{\mathrm{ch}, 3}=30 \mathrm{~mm}$. The mean thickness between the two surfaces is $H=40 \mathrm{~mm}$. In this paper, we consider only one realisation of the two surfaces. The cylinder is centered at the point of coordinates $C=(0,-0.5 H)$. Fig. 2 plots the heights (in $\mathrm{mm}$ ) versus their abscissa (in $\mathrm{mm}$ ). The medium relative permittivities are $\epsilon_{\mathrm{r}, 0}=1, \epsilon_{\mathrm{r}, 1}=2, \epsilon_{\mathrm{r}, 2}=7$, and $\epsilon_{\mathrm{r}, 3}=4$. As we will focus on GPR applications, we consider a Thorsos wave at normal incidence $\left(\theta_{\text {inc }}=0^{\circ}\right)$, of frequency $f=2 \mathrm{GHz}$ and whose attenuation parameter is $g=L / 6$. The number of points $n_{i}(i=\{1,2,3\})$ per wavelength is 10 and the wavelength in vaccuum is $\lambda_{0}=150 \mathrm{~mm}$.

To test the convergence of the GPILE algorithm, we plot the scattered field and calculate the residual relative error (RRE). In fact, the MoM with the classical LU inversion contains an infinite number of interactions between the three scatterers, whereas for GPILE we only consider a limited number of interactions. We have defined two types of interactions, those called principal (between the upper surface and the lower surface or the object) and those called secondary (between the object and the lower surface). In this way, the orders $p$ and $q$ of GPILE are the principal order and the secondary order, respectively. The residual relative error is calculated by

$$
\mathrm{RRE}=\frac{\operatorname{norm}\left(\boldsymbol{X}-\boldsymbol{X}_{\mathrm{LU}}\right)}{\operatorname{norm}\left(\boldsymbol{X}_{\mathrm{LU}}\right)}
$$

where the norm of a vector of components $\boldsymbol{X}_{i}$ and of length
$N$ is given by

$$
\operatorname{norm}(\boldsymbol{X})=\sum_{i=1}^{N}\left|\boldsymbol{X}_{i}\right|^{2} .
$$

The vector $\boldsymbol{X}$ can represent the surface currents $\psi_{i}$ or their normal derivatives $\partial \psi_{i} / \partial n_{i}$ calculated by GPILE on all surfaces. In the context of this study, $\boldsymbol{X}$ will rather represent the scattered field. The LU label means that the vector is calculated by the LU inversion algorithm.

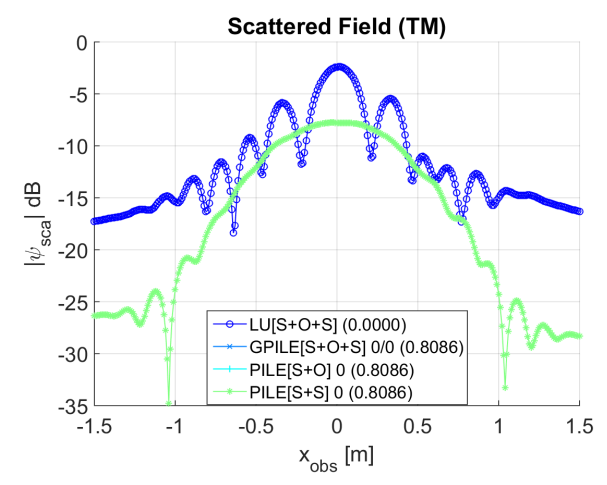

Fig. 3. Modulus of the scattered field in decibel scale $\left(10 \log _{10}\left|\psi_{\text {sca }}\right|\right)$ versus the observation abscissa $x_{\text {obs }} \in[-L / 2 ; L / 2]$, for an observation height $z_{\mathrm{obs}}=470 \mathrm{~mm}$. The frequency is $f=2 \mathrm{GHz}$ and $P=0$.

We present the following figures where we compare both the GPILE and LU methods as well as the PILE methods for the cases of two superimposed surfaces or an object buried under a surface. This allows us to study the performance of GPILE and also to show the contributions of the two buried scatterers. We present the moduli of the scattered fields in decibels $\left(10 \log _{10}\left|\psi_{\text {sca }}\right|\right)$ with respect to the observation abscissa $\left(x_{\mathrm{obs}} \in[-L / 2 ; L / 2]\right)$. In the legend, $\mathrm{S}+\mathrm{O}+\mathrm{S}$ represents the case of an object buried between two surfaces, while $\mathrm{S}+\mathrm{O}$ represents an object buried under a surface, and $\mathrm{S}+\mathrm{S}$ the case of two superimposed surfaces. Then, we have the orders $P$ and $Q$ (or only $P$ for PILE). The number in parenthesis is the RRE with respect to the reference LU method.

We first look at the impact of the principal order $p$. Thus, in Fig. 3, the PILE and GPILE methods are presented for $P=0$. We observe that the scattered fields calculated by the three methods are exactly the same, because at this order, the three methods calculate only the first echo, with account only the upper surface. In Fig. 4 (a)-(b), $P=1$, differences between the three methods appear because for the PILE methods, the upper surface only interacts with the lower surface or the object, but for GPILE, the upper surface interacts with both the lower surface and the object. This makes it possible to highlight the presence of the object between the two surfaces. In Fig. 4 (c)-(d), with $P=2$, we observe a perfect agreement between the LU method and the GPILE method when $Q=1$, which shows that $P=2$ is sufficient to take account all the significant contributions. Note also that $P=2$ means that the wave makes two round trips between the upper surface and the lower surface or the object. 

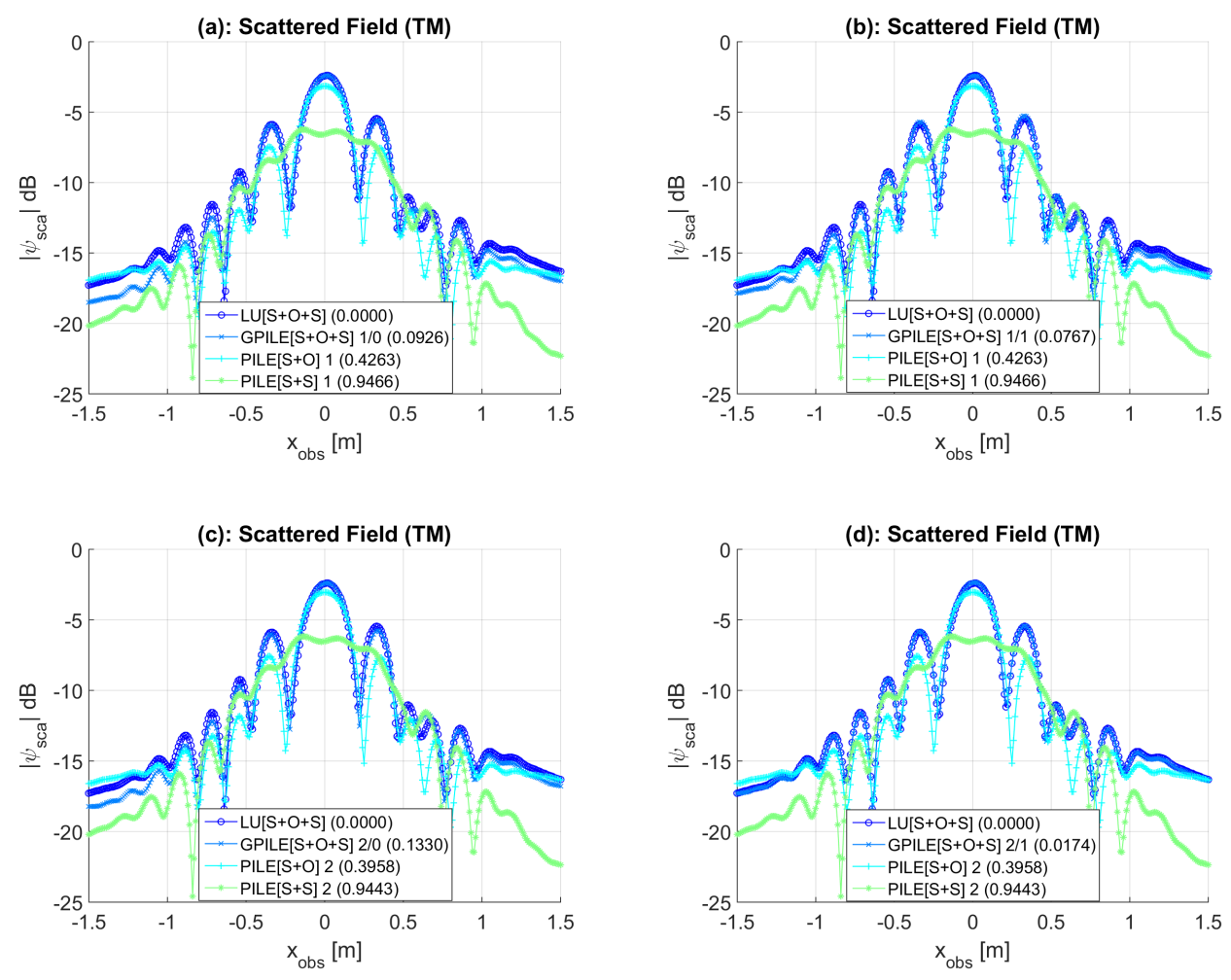

Fig. 4. Modulus of the scattered field in decibel scale $\left(10 \log _{10}\left|\psi_{\text {sca }}\right|\right)$ versus the observation abscissa $x_{\text {obs }} \in[-L / 2 ; L / 2]$ and for an observation height $z_{\mathrm{obs}}=470 \mathrm{~mm}$. The frequency is $f=2 \mathrm{GHz}$ and the orders $P$ and $Q$ vary.
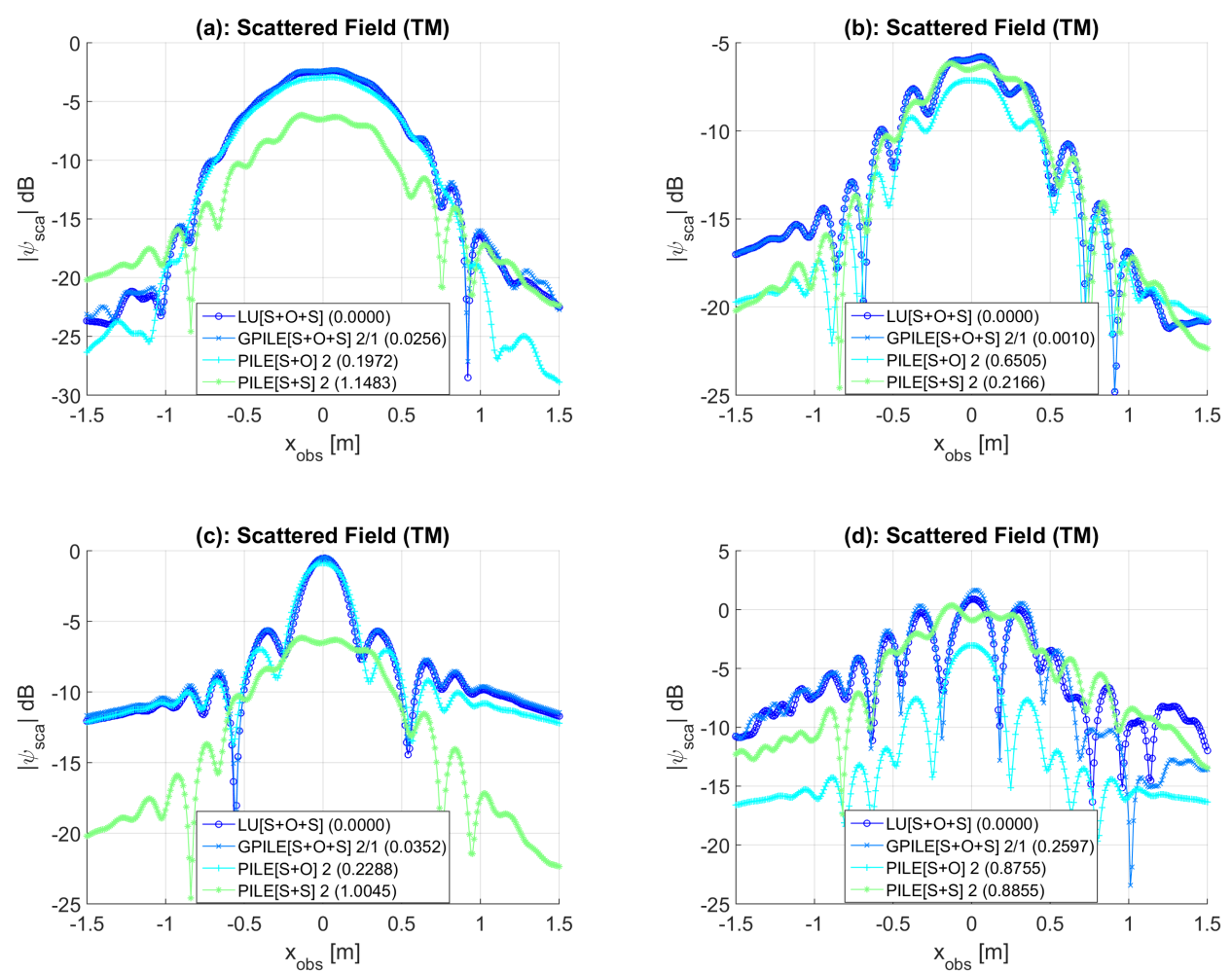

Fig. 5. Modulus of the scattered field in decibel scale $\left(10 \log _{10}\left|\psi_{\text {sca }}\right|\right)$ versus the observation abscissa $x_{\text {obs }} \in[-L / 2 ; L / 2]$ and for an observation height $z_{\mathrm{obs}}=470 \mathrm{~mm}$. The frequency is $f=2 \mathrm{GHz}$ and the orders $P=2$ and $Q=1$. (a) Cylinder with $a=1000$ mm and $b=10 \mathrm{~mm}$. (b) Cylinder with $a=b=10 \mathrm{~mm}$. (c) The object is a perfect conductor, with $a=100 \mathrm{~mm}$ and $b=10 \mathrm{~mm}$. (c) The lower surface is a perfect conductor, with $a=100 \mathrm{~mm}$ and $b=10 \mathrm{~mm}$. 
To evaluate the impact of the secondary order $q$, several numerical simulations, that we do not present here, show that $Q=0$ or 1 is sufficient for the convergence of the algorithm when $P \geq 1$ and that the higher orders of $q$ do not have a significant impact on the precision of GPILE. Besides, $Q=0$ means that the wave does not perform a round trip between the object and the lower surface, and vice versa. However, even for $Q=0$, there are interactions between these two scatterers via $\bar{Z}_{12}^{\prime}$ and $\bar{Z}_{13}^{\prime}$ as shown by the relations (15) and (18), respectively.

We show in figures 5 the impact of some parameters of the object on the scattered field. Fig. 5 (a) plots the case of a large elliptical cylinder with semi-major axis $a=1000 \mathrm{~mm}$ while Fig. 5(b) plots the case of a small elliptical cylinder with semimajor axis $a=10 \mathrm{~mm}$. The semi-minor axis is $b=10 \mathrm{~mm}$ for the two cylinders. We can see on the levels of curves as well as on the RRE that the contribution of the object varies proportionally to its size. We also observe that the scattered field of an object buried between two rough surfaces tends towards that of two rough surfaces when the object becomes smaller. Likewise, regarding the relative permittivities, we show in Fig. 5(c)-(d) that the contributions of the object or the lower surface are much more important when these two scatterers are perfect conductors. Remark also that, in all these cases, the GPILE method converges for $P=2$ and $Q=1$, despite changing parameters. This remark also hold for the simulations presented in section $\mathrm{V}$, in which the frequency band is $[0.2-6.0] \mathrm{GHz}$. In the following, we consider the GPILE method for $P=2$ and $Q=1$ as a reference for the study of electromagnetic scattering by an object buried between two random rough surfaces.

In addition, it has already been established that the scattered field in the case of two superimposed rough interfaces depends on the geometry and the physical parameters, such as the permittivities of the media, the statistical parameters of the interfaces and the thickness between these interfaces. Turning now to the study of an object buried between two rough surfaces, several simulations that we do not present here show that in addition to the surface parameters, the scattered field also depends on the parameters of the object such as its size, shape, position and permittivity. In this way, the sensitivity of the scattered field to the variation of these parameters could allow the characterization of the object in an inverse problem.

\section{FREQUENCY AND TIME RESPONSE RESUlTS}

In this section, PILE and GPILE methods are used to provide simulations of GPR data for an object buried between two random rough surfaces. To compute the GPR responses, both methods are performed on $N_{f}$ equaly spaced frequencies covering the GPR band.

A Ricker pulse is considered as an input signal [24]. In the time domain, it is defined as

$$
s(t)=\left(2 \pi^{2} f_{c}^{2} t^{2}-1\right) \exp \left(-\pi^{2} f_{c}^{2} t^{2}\right)
$$

In the Fourier domain, it is defined as

$$
\widehat{s}(f)=-\frac{2 f^{2}}{f_{c}^{2} \sqrt{\pi}} \exp \left(-\frac{f^{2}}{f_{c}^{2}}\right), f \geq 0
$$

The absolute value of the spectrum is maximum for $f=f_{c}$ and the value of the maximum is $\widehat{s}_{0}=2 /\left(e f_{c} \sqrt{\pi}\right)$. For our simulations, we take $f_{c}=2 \mathrm{GHz}$. Fig. 6 plots the Fourier transform versus the frequency $f$ and the signal versus the time $t$. As shown in Fig. 6(a), the signal is Hermitian, i.e., $\widehat{s}(-f)^{*}=\widehat{s}(+f)$, where the symbol $*$ stands for the complex conjugate. The number of frequencies is $N_{f}=117$ and the band is $[0.2-6.0] \mathrm{GHz}$. To have a better resolution in the frequency domain, we applied the zero-padding technique to increase $N_{f}$ to $2^{12}=4096$. Thus, the sampling step in the frequency domain is $\Delta f=0.00142 \mathrm{GHz}$.
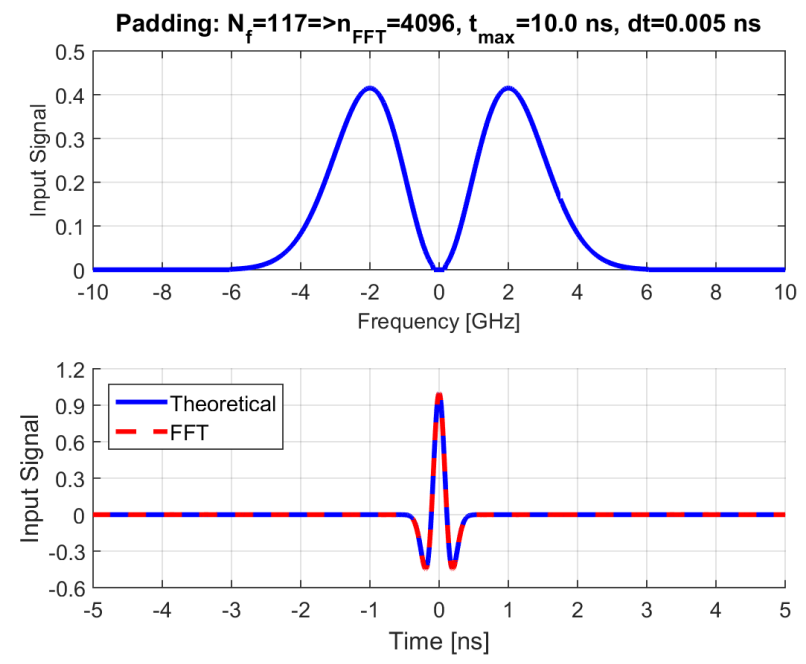

Fig. 6. (a) The Fourier transform of the signal versus the frequency $f$ (in $\mathrm{GHz}$ ). (b) Signal versus the time $t$ (in $\mathrm{ns}$ ).

Fig. 7 plots the frequency response of the modulus of the scattered field versus the frequency $f$. The simulations parameters are the same as in Fig. 4, exept the thickness $H=240$ $\mathrm{mm}$ and the center of the cylinder $C=(0,-0.375 H)$. The observation abscissa and height are $x_{\mathrm{obs}}=0$ and $z_{\mathrm{obs}}=470$ $\mathrm{mm}$, respectively. Fig. 7(a) shows the input signal and the scattered field. In Fig. 7(b), the GPILE and PILE methods give the same results because for $P=0$, the three methods are calculated for the first echo only i.e. the incident wave interacts only with the upper surface. In Figs. 7(c)-(d), the difference between the three methods appears because for the PILE methods, the upper surface interacts only with the lower surface or the object, but for the GPILE method, the upper surface interacts with both the lower surface and the object. On the other hand, the non-remarquable difference between these two figures shows that the third echo is weak. We will see later that this is the case.

Fig. 8 plots the time responses of the scattered field. The simulations parameters are the same as in Fig. 7. For smooth interfaces, the first three echoes of the lower surface arise at the times $T_{1}, T_{2, \mathrm{~S}}$ and $T_{3, \mathrm{~S}}$ defined as :

$$
\left\{\begin{array}{l}
T_{1}=2 z_{0} / c \\
T_{2, \mathrm{~S}}=T_{1}+2 H \operatorname{Re}\left(\sqrt{\epsilon_{\mathrm{r}, 1}}\right) \\
T_{3, \mathrm{~S}}=T_{1}+4 H \operatorname{Re}\left(\sqrt{\epsilon_{\mathrm{r}, 1}}\right)
\end{array}\right.
$$



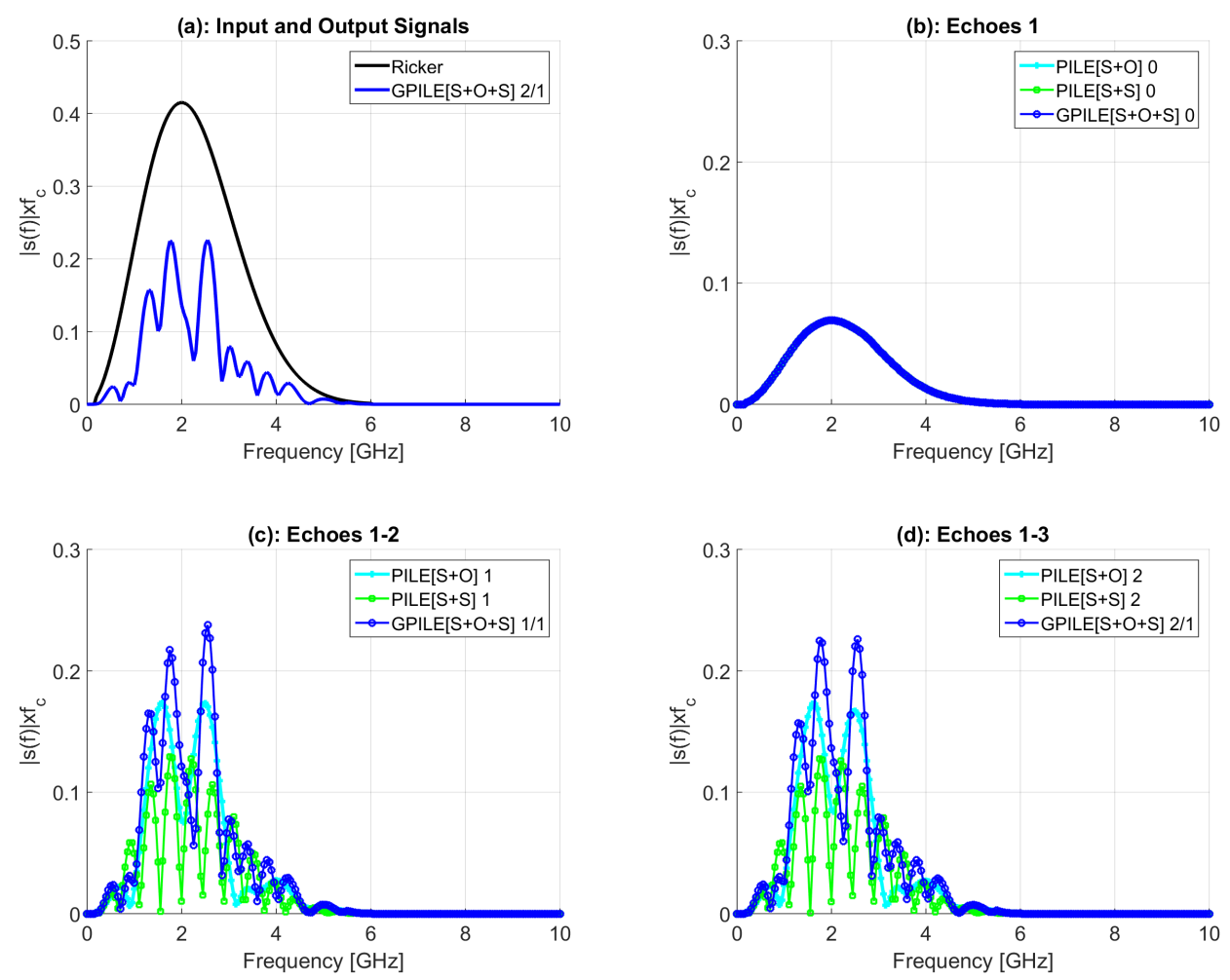

Fig. 7. Frequency responses of the modulus of the scattered field. $x_{o b s}=0, z_{o b s}=470 \mathrm{~mm}$. (a) Input and Output signals. (b) First echo $(P=0)$. (c) First two echoes $(P=1)$. (d) First three echoes $(P=2)$.
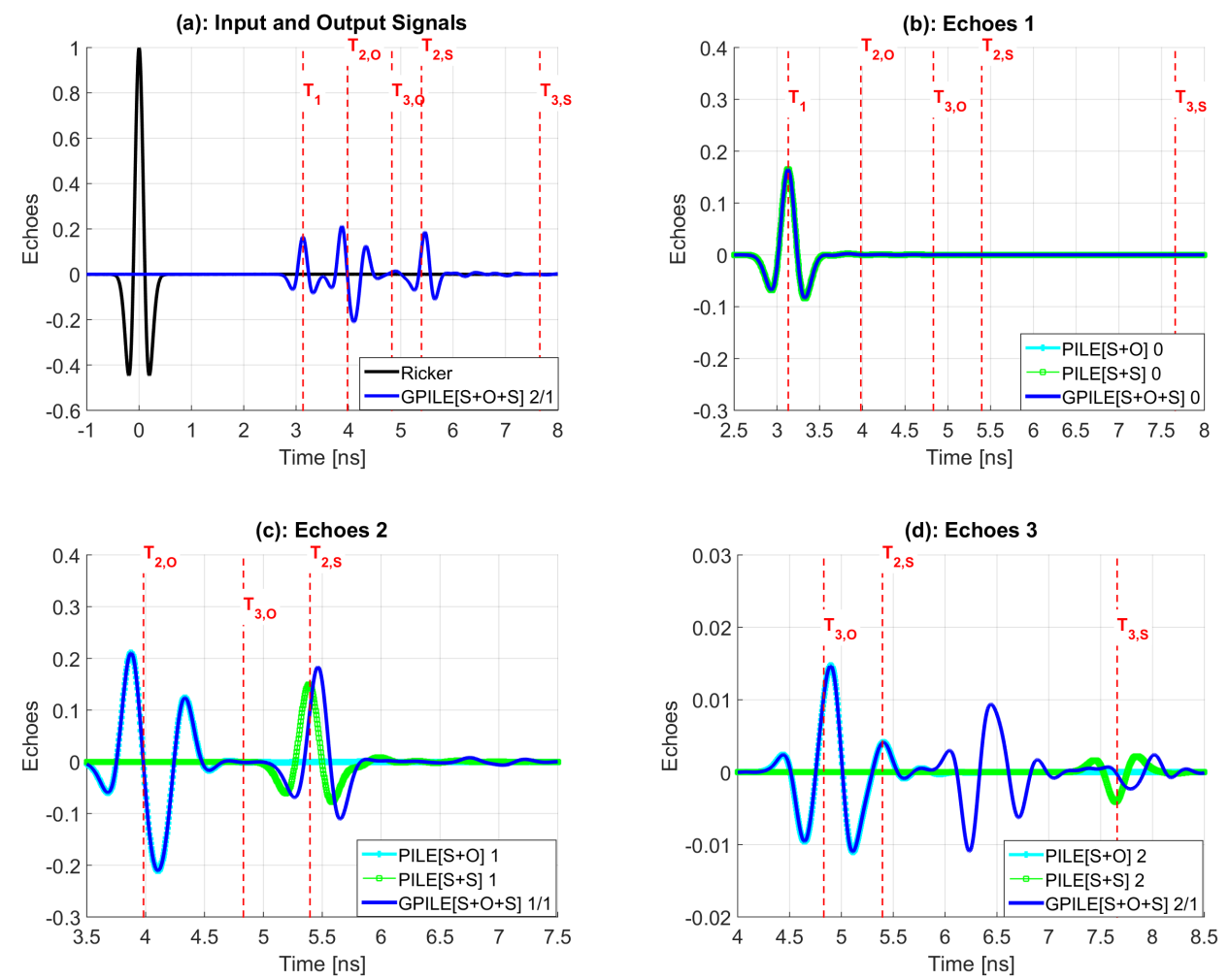

Fig. 8. Time responses of the modulus of the scattered field. $x_{\mathrm{obs}}=0, z_{\mathrm{obs}}=470 \mathrm{~mm}$. (a) Input and Output signals. (b) First echoes $(P=0)$. (c) Second echoes $(P=1)$. (d) Third echoes $(P=2)$. 
where $H$ is the thickness of the layer. We can obtain the second and third echo times for the object by replacing $H$ by $H_{12}$ in (31), where $H_{12}$ is the depth of the object. Those echoes are written $T_{2, \mathrm{O}}$ and $T_{3, \mathrm{O}}$. Note that the depth of the object can be calculated in three different ways, first relative to the center of the object, second relative to the upper vertex, and finally relative to the lower vertex. Each way of calculating this depth gives a different result. In this paper we will focus on calculating the depth of the object from the center.

Remember that these echoes are calculated by the PILE methods. Regarding the GPILE method, the second and third echoes result from multiple scattering between the object and the lower surface in the medium $\Omega_{1}$. Thus, for $P=1$ and $Q=0$, the second echo is made up of two contributions: one from the object and the other one from the lower surface. Each of these contributions has two components : one direct and the other one indirect. For the object for example, we have seen in relation (15) that the contribution matrix is composed by the direct contribution, represented by $\bar{Z}_{12}$ (taking account for the wave coming from the scattering on the object of the wave transmitted by the upper surface) and the indirect contribution represented by $\overline{\boldsymbol{Z}}_{12}^{\prime}$ (taking account for the wave coming from the scattering on the object of the wave coming from the scattering on the lower surface of the wave transmitted by the upper surface). For the lower surface, the direct and indirect contributions are highlighted in relation (18) and represented by the contribution matrices $\bar{Z}_{13}$ and $\overline{\boldsymbol{Z}}_{13}^{\prime}$, respectively.

Moreover, the direct contribution echoes of the object and of the lower surface arise at different times, because for the first one the wave goes back and forth to the object while for the second one the wave goes back and forth to the lower surface. In fact, the indirect contribution echoes of the two scatterers arise at the same time (see Ind. Object and Ind. Lower Surf. in Fig. 9). For the object, the wave goes first to the lower surface, then passes through the object before returning to the upper surface. For the lower surface, the wave passes through the same path but in the opposite direction. Theoretically, the indirect contribution echoes arrive at the same time as the direct contribution echo from the lower surface. However, in practice a time lag will be created for the indirect contribution echoes because of the scattering from the object. For $Q=1$, it suffices to add the time of a round trip between the object and the lower surface, given by $T_{\mathrm{OS}}=T_{2, \mathrm{~S}}-T_{2, \mathrm{O}}$.

In Fig. 8, PILE methods show the impact of the object and of the lower surface while GPILE method takes also account the multiple interactions between the two buried scatterers. As can be seen in figures $8(\mathrm{a})$ and $8(\mathrm{~b})$, the first echo is correlated to the input signal. Fig. 8(c) shows that the second echo of the object has two peaks between $[3.5-4.5] \mathrm{ns}$. The first peak comes from the signal emanating directly from the antenna while the second peak comes from the closed shape of the object and the roughness of the upper surface. The second echo of the lower surface has a peak between $[5-5.5]$ ns. This peak is followed by a few low intensity peaks linked to the roughness of the two surfaces.

The times calculated for the second echoes from the two buried scatterers are consistent with the observed times. We calculated the depth of the object in relation to its center and we obtained $T_{2, \mathrm{O}}=3.98 \mathrm{~ns}$, which corresponds to the point of zero amplitude (inflexion point). However, if we calculated the depth from the upper vertex, we would have $T_{2, \mathrm{O}}=3.88 \mathrm{~ns}$, which corresponds to the maximum amplitude peak. On the other hand, if we calculated the depth from the lower vertex, we would have $T_{2, \mathrm{O}}=4.07 \mathrm{~ns}$, which corresponds to the minimum amplitude peak.

The second echo for GPILE $1 / 1$ is composed of the sum of the direct and indirect contribution echoes of the two buried scatterers, and one interaction between these scatterers. Observe that GPILE and PILE(S+O) concide around $T_{2, \mathrm{O}}$ as shown in Figs 8(c), because the direct contribution echo for the object for GPILE is exactly PILE(S+O). However, for the lower surface, there is a time lag between $\operatorname{PILE}(\mathrm{S}+\mathrm{S})$ and GPILE around $T_{2, \mathrm{~S}}$ due to the scattering on the object. There is also a difference of amplitude between PILE $(\mathrm{S}+\mathrm{S})$ and GPILE around $T_{2, \mathrm{~S}}$, because the direct contribution echo of the lower surface arrives at the same time as the indirect contribution echoes of the two buried scatterers, and as $Q=1$, one must add the contribution of one round trip of the wave between the object and the lower surface for the direct contribution of the object. This last contribution also justifies the difference in the amplitude between GPILE $1 / 0$ and GPILE $1 / 1$ around $T_{2, \mathrm{~S}}$ (see Fig. 9). The contribution of the lower surface for $Q=1$ arives at around $T_{2, \mathrm{~S}}+T_{\mathrm{OS}}=6.81 \mathrm{~ns}$ and can be observed in Fig. 8(c). This contribution justifies the difference in amplitude between GPILE $1 / 0$ and GPILE $1 / 1$ between $[6.5-7.5]$ ns as shows Fig. 9.

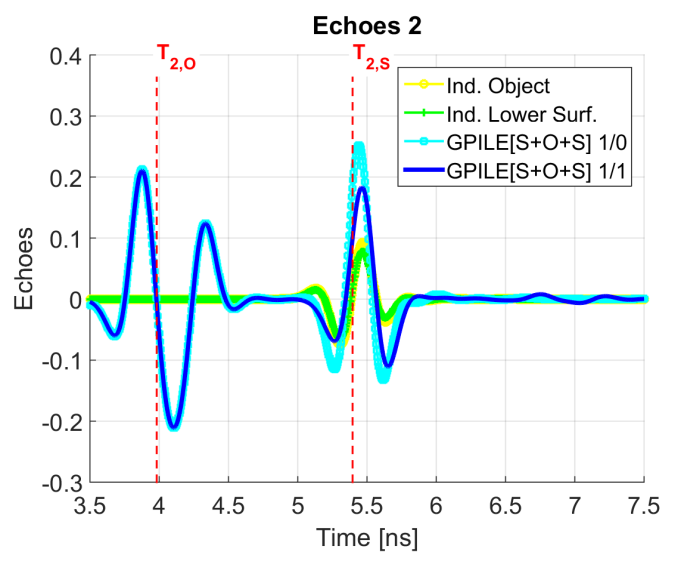

Fig. 9. Time response of the modulus of the scattered field: Direct and Indirect contributions. $x_{\mathrm{obs}}=0, z_{\mathrm{obs}}=470 \mathrm{~mm}$.

We now want to analyze the different contributions that make up the third echo of GPILE. To do this, remember that the second echo is made up of four contributions, namely, a direct contribution and another indirect for each of the buried scatterers. Each of the four contributions of the second echo will create four contributions for the third echo, making a total of 16 contributions for the third echo, for a fixed value of $Q$. We are first interested in the cases of third echoes which do not contain secondary interactions i.e. for which $Q=0$. We have seen that for the second echo, the direct contribution of the object arrives before its indirect contribution, which arrives at the same time as the two contributions of the lower surface. 
Thus, we can group the 16 contribution echoes that make up the third echo of GPILE into three classes. In the first class, we have the echo which involves only the direct contribution of the object, both for the second echo and for the third echo. This echo arises at the time $T_{\mathrm{SO}}+T_{2, \mathrm{O}}=4.64 \mathrm{~ns}$ where $T_{\mathrm{SO}}$ is the time of a round trip of the wave between the upper surface and the object, which is why $\operatorname{PILE}(\mathrm{S}+\mathrm{O})$ coincides with GPILE between $[4-6] \mathrm{ns}$ as shown in Fig. 8(d). In the second class, we have all the other echoes that involve the direct contribution of the object, whatever the contribution of the lower surface. These echoes are six and arise at the time $T_{\mathrm{SO}}+T_{2, \mathrm{~S}}=6.15 \mathrm{~ns}$ and can be observed in the interval $[6-7]$ ns. In the third class, we have all the echoes which do not involve the direct contribution of the object, and this whatever the contribution of the lower surface. These echoes are nine and arrive at the time $T_{\mathrm{SS}}+T_{2, \mathrm{~S}}=T_{3, \mathrm{~S}}=7.65 \mathrm{~ns}$ where $T_{\mathrm{SS}}$ is the time of a round trip of the wave between the upper surface and the lower surface, and can be observed in the interval $[7-8.5]$ ns.

Note that for $Q=1$, it suffices to add the time of a round trip of the wave between the object and the lower surface, and recalculate all the contribution echo times. However, we cannot detail these calculations here as long as these contributions are very weak as we have already underlined.

\section{CONCLUSION}

In this paper, the GPILE method has been developed to rigorously calculate the frequency and time responses of the scattered field for an object buried between two random rough surfaces. This method have a straightforward physical interpretation and allow us to distinguish the primary echo of the upper surface, the multiple echoes coming from the lower surface and those arising from the object. The GPILE method is applied to simulate the Ground Penetrating Radar signal at nadir in both frequency and time domains. The simulated signal was used to detect the presence of the object buried between the two random rough surfaces.

\section{APPENDIX A}

\section{SUb-MATRICES OF THE IMPEDANCE MATRIX}

In the case of electromagnetic wave scattering from an object buried between two rough surfaces, the sub-matrices of the impedance matrix are:

$$
\begin{aligned}
& \overline{\boldsymbol{Z}}_{11}=\left(\begin{array}{cc}
\overline{\boldsymbol{A}}_{1} & \overline{\boldsymbol{B}}_{1} \\
\overline{\boldsymbol{C}}_{1} & \rho_{10} \overline{\boldsymbol{D}}_{1}
\end{array}\right), \quad \overline{\boldsymbol{Z}}_{21}=\left(\begin{array}{cc}
\overline{\mathbf{0}} & \overline{\mathbf{0}} \\
\overline{\boldsymbol{A}}_{21} & \overline{\boldsymbol{B}}_{21}
\end{array}\right), \\
& \overline{\boldsymbol{Z}}_{31}=\left(\begin{array}{cc}
\overline{\mathbf{0}} & \overline{\mathbf{0}} \\
\overline{\boldsymbol{A}}_{31} & \overline{\boldsymbol{B}}_{31}
\end{array}\right), \quad \overline{\boldsymbol{Z}}_{12}=\left(\begin{array}{cc}
\overline{\boldsymbol{A}}_{12} & \rho_{10} \overline{\boldsymbol{B}}_{12} \\
\overline{\mathbf{0}} & \overline{\mathbf{0}}
\end{array}\right), \\
& \overline{\boldsymbol{Z}}_{22}=\left(\begin{array}{cc}
\overline{\boldsymbol{A}}_{2} & \overline{\boldsymbol{B}}_{2} \\
\overline{\boldsymbol{C}}_{2} & \rho_{21} \overline{\boldsymbol{D}}_{2}
\end{array}\right), \quad \overline{\boldsymbol{Z}}_{32}=\left(\begin{array}{cc}
\overline{\boldsymbol{A}}_{32} & \overline{\boldsymbol{B}}_{32} \\
\overline{\mathbf{0}} & \overline{\mathbf{0}}
\end{array}\right), \\
& \overline{\boldsymbol{Z}}_{13}=\left(\begin{array}{cc}
\overline{\boldsymbol{A}}_{13} & \rho_{10} \overline{\boldsymbol{B}}_{13} \\
\overline{\mathbf{0}} & \overline{\mathbf{0}}
\end{array}\right), \overline{\boldsymbol{Z}}_{23}=\left(\begin{array}{cc}
\overline{\boldsymbol{A}}_{23} & \overline{\boldsymbol{B}}_{23} \\
\overline{\mathbf{0}} & \overline{\mathbf{0}}
\end{array}\right), \\
& \overline{\boldsymbol{Z}}_{33}=\left(\begin{array}{cc}
\overline{\boldsymbol{A}}_{3} & \overline{\boldsymbol{B}}_{3} \\
\overline{\boldsymbol{C}}_{3} & \rho_{31} \overline{\boldsymbol{D}}_{3}
\end{array}\right) .
\end{aligned}
$$

where the matrices $\overline{\boldsymbol{Z}}_{i i}$ are the self-impedance matrices of the surfaces $S_{i}(i=1,2,3)$, while the matrices $\overline{\boldsymbol{Z}}_{i j}$ are the coupling matrices between interfaces $S_{i}$ and $S_{j}(i \neq j)$.
Since an interface cannot be illuminated from both above and below (from the outside and the inside for an object) by the same scatterer, this mathematically results in the fact that the upper blocks of matrices $\bar{Z}_{21}$ and $\bar{Z}_{31}$ are zero because the upper surface is illuminated only by the object and the lower surface in $\Omega_{1}$. Likewise, the lower blocks of $\overline{\boldsymbol{Z}}_{12}$ and $\overline{\boldsymbol{Z}}_{32}$ are zero because the object is illuminated only from the outside. Finally, in the matrices $\overline{\boldsymbol{Z}}_{13}$ and $\overline{\boldsymbol{Z}}_{23}$, the lower blocks are zero because the lower surface is illuminated only in $\Omega_{1}$.

The elements of the sub-matrices of the impedance matrix are given by

$$
\begin{aligned}
& \overline{\boldsymbol{A}}_{1, m n}=\left\{\begin{array}{l}
-\frac{j k_{0} v_{n}\left|\Delta_{n}\right|}{4} \frac{H_{1}^{(1)}\left(k_{0}\left\|\boldsymbol{r}_{n}-\boldsymbol{r}_{m}\right\|\right)}{\left\|\boldsymbol{r}_{n}-\boldsymbol{r}_{m}\right\|} \times \\
{\left[\gamma_{n}\left(x_{n}-x_{m}\right)-\left(z_{n}-z_{m}\right)\right] \text { for } m \neq n,} \\
+\frac{1}{2}-\frac{v_{n}\left|\Delta_{n}\right|}{4 \pi} \frac{\gamma^{\prime}\left(x_{n}\right)}{1+\gamma^{2}\left(x_{n}\right)}, \text { for } m=n .
\end{array}\right. \\
& \overline{\boldsymbol{B}}_{1, m n}=\frac{j\left|\Delta_{n}\right| \sqrt{1+\gamma_{n}^{2}}}{4}\left\{\begin{array}{l}
{\left[1+\frac{2 j}{\pi} \ln \left(0.164 k_{0} \sqrt{1+\gamma_{n}^{2}} \Delta_{n}\right)\right],} \\
\text { for } m=n, \\
H_{0}^{(1)}\left(k_{0}\left\|\boldsymbol{r}_{n}-\boldsymbol{r}_{m}\right\|\right), \text { for } m \neq n .
\end{array}\right. \\
& \overline{\boldsymbol{C}}_{1, m n}=\left\{\begin{array}{l}
-\frac{j k_{1} v_{n}\left|\Delta_{n}\right|}{4} \frac{H_{1}^{(1)}\left(k_{1}\left\|\boldsymbol{r}_{n}-\boldsymbol{r}_{m}\right\|\right)}{\left\|\boldsymbol{r}_{n}-\boldsymbol{r}_{m}\right\|} \times \\
{\left[\gamma_{n}\left(x_{n}-x_{m}\right)-\left(z_{n}-z_{m}\right)\right], \text { for } m \neq n} \\
-\frac{1}{2}-\frac{v_{n}\left|\Delta_{n}\right|}{4 \pi} \frac{\gamma^{\prime}\left(x_{n}\right)}{1+\gamma^{2}\left(x_{n}\right)}, \text { for } m=n .
\end{array}\right. \\
& \overline{\boldsymbol{D}}_{1, m n}=\frac{j\left|\Delta_{n}\right| \sqrt{1+\gamma_{n}^{2}}}{4}\left\{\begin{array}{l}
{\left[1+\frac{2 j}{\pi} \ln \left(0.164 k_{1} \sqrt{1+\gamma_{n}^{2}} \Delta_{n}\right)\right],} \\
\text { for } m=n, \\
H_{0}^{(1)}\left(k_{1}\left\|\boldsymbol{r}_{n}-\boldsymbol{r}_{m}\right\|\right), \text { for } m \neq n .
\end{array}\right.
\end{aligned}
$$

where $\left(x_{n}, z_{n}\right) \in S_{i},\left(x_{m}, z_{m}\right) \in S_{i}, \gamma=d z / d x, \| \boldsymbol{r}_{n}-$ $\boldsymbol{r}_{m} \|=\sqrt{\left(x_{n}-z_{n}\right)^{2}+\left(x_{m}-z_{m}\right)^{2}}, v=\hat{\boldsymbol{n}} \cdot \hat{\boldsymbol{z}}$ is the direction of the normal to the surface, $\left|\Delta_{n}\right|$ is the discretization step size, $H_{0}^{(1)}$ is a zero-order Hankel function of the first kind and $H_{1}^{(1)}$ its derivative.

The elements of the matrices $\overline{\boldsymbol{A}}_{2}, \overline{\boldsymbol{B}}_{2}, \overline{\boldsymbol{C}}_{2}$ and $\overline{\boldsymbol{D}}_{2}$ are obtained from those of $\overline{\boldsymbol{A}}_{1}, \overline{\boldsymbol{B}}_{1}, \overline{\boldsymbol{C}}_{1}$ and $\overline{\boldsymbol{D}}_{1}$, in which the wave numbers $\left\{k_{0}, k_{0}, k_{1}, k_{1}\right\}$ are replaced by $\left\{k_{1}, k_{1}, k_{2}, k_{2}\right\}$ respectively. In the same way, we obtain the elements of $\overline{\boldsymbol{A}}_{3}, \overline{\boldsymbol{B}}_{3}, \overline{\boldsymbol{C}}_{3}$ and $\overline{\boldsymbol{D}}_{3}$ by replacing the wave numbers by $\left\{k_{1}, k_{1}, k_{3}, k_{3}\right\}$.

Moreover, the elements of the coupling matrices between the three interfaces are given $\forall i, i^{\prime}=1,2,3$, by:

$$
\begin{gathered}
\overline{\boldsymbol{A}}_{i i^{\prime}, n m}=-\frac{j k_{1} v_{i, n}\left|\Delta_{i, n}\right|}{4} \frac{H_{1}^{(1)}\left(k_{1}\left\|\boldsymbol{r}_{i, n}-\boldsymbol{r}_{i^{\prime}, m}\right\|\right)}{\left\|\boldsymbol{r}_{i, n}-\boldsymbol{r}_{i^{\prime}, m}\right\|} \times \\
{\left[\gamma_{i, n}\left(x_{i, n}-x_{i^{\prime}, m}\right)-\left(z_{i, n}-z_{i^{\prime}, m}\right)\right]}
\end{gathered}
$$

and

$$
\overline{\boldsymbol{B}}_{i i^{\prime}, n m}=\frac{j\left|\Delta_{i, n}\right| \sqrt{1+\gamma_{i, n}^{2}}}{4} H_{0}^{(1)}\left(k_{1}\left\|\boldsymbol{r}_{i, n}-\boldsymbol{r}_{i^{\prime}, m}\right\|\right) .
$$

The indexes $i, n$ and $i^{\prime}, m$ indicate that the points considered are on two different surfaces. 


\section{APPENDIX B}

\section{RESOlutions of the System $\overline{\boldsymbol{Z}} \boldsymbol{X}=\boldsymbol{b}$ B The} Substitution Method

Consider the linear system obtained by the MoM for the electromagnetic scattering by an object buried between two surfaces (or two objects buried under a surface):

$$
\left\{\begin{array}{l}
\overline{\boldsymbol{Z}}_{11} \boldsymbol{X}_{1}+\overline{\boldsymbol{Z}}_{21} \boldsymbol{X}_{2}+\overline{\boldsymbol{Z}}_{31} \boldsymbol{X}_{3}=\boldsymbol{b}_{1} \\
\overline{\boldsymbol{Z}}_{12} \boldsymbol{X}_{1}+\overline{\boldsymbol{Z}}_{22} \boldsymbol{X}_{2}+\overline{\boldsymbol{Z}}_{32} \boldsymbol{X}_{3}=\mathbf{0} \\
\overline{\boldsymbol{Z}}_{13} \boldsymbol{X}_{1}+\overline{\boldsymbol{Z}}_{23} \boldsymbol{X}_{2}+\overline{\boldsymbol{Z}}_{33} \boldsymbol{X}_{3}=\mathbf{0}
\end{array}\right.
$$

We have two possibilities to solve the system.

(i) The expression of $\boldsymbol{X}_{3}$ is introduced in the second equation, which makes it possible to write $\boldsymbol{X}_{2}$ as a function of $\boldsymbol{X}_{1}$ so that

$$
\begin{aligned}
\boldsymbol{X}_{2}= & \left(\overline{\boldsymbol{I}}-\overline{\boldsymbol{Z}}_{22}^{-1} \overline{\boldsymbol{Z}}_{32} \overline{\boldsymbol{Z}}_{33}^{-1} \overline{\boldsymbol{Z}}_{23}\right)^{-1} \overline{\boldsymbol{Z}}_{22}^{-1} \times \\
& \left(\overline{\boldsymbol{Z}}_{12}^{\prime}-\overline{\boldsymbol{Z}}_{12}\right) \boldsymbol{X}_{1}
\end{aligned}
$$

thus

$$
\boldsymbol{X}_{2}=-\left(\sum_{q=0}^{Q} \overline{\boldsymbol{M}}_{c, 32}^{q}\right) \overline{\boldsymbol{Z}}_{22}^{-1}\left(\overline{\boldsymbol{Z}}_{12}-\overline{\boldsymbol{Z}}_{12}^{\prime}\right) \boldsymbol{X}_{1},
$$

where

$$
\overline{\boldsymbol{M}}_{c, 32}=\overline{\boldsymbol{Z}}_{22}^{-1} \overline{\boldsymbol{Z}}_{32} \overline{\boldsymbol{Z}}_{33}^{-1} \overline{\boldsymbol{Z}}_{23} .
$$

Then, the expressions of $\boldsymbol{X}_{2}$ are introduced into the third equation, which makes it possible to write $\boldsymbol{X}_{3}$ as a function of $\boldsymbol{X}_{1}$ so that

$$
\begin{aligned}
\boldsymbol{X}_{3} & =\overline{\boldsymbol{Z}}_{33}^{-1} \overline{\boldsymbol{Z}}_{23}\left(\sum_{q=0}^{Q} \overline{\boldsymbol{M}}_{c, 32}^{q}\right) \overline{\boldsymbol{Z}}_{22}^{-1}\left(\overline{\boldsymbol{Z}}_{12}-\overline{\boldsymbol{Z}}_{12}^{\prime}\right) \boldsymbol{X}_{1} \\
& -\overline{\boldsymbol{Z}}_{33}^{-1} \overline{\boldsymbol{Z}}_{13} \boldsymbol{X}_{1} .
\end{aligned}
$$

The expressions of $\boldsymbol{X}_{2}$ and $\boldsymbol{X}_{3}$ are introduced into the first equation, rewritten as

$$
\boldsymbol{X}_{1}+\overline{\boldsymbol{Z}}_{11}^{-1} \overline{\boldsymbol{Z}}_{21} \boldsymbol{X}_{2}+\overline{\boldsymbol{Z}}_{11}^{-1} \overline{\boldsymbol{Z}}_{31} \boldsymbol{X}_{3}=\overline{\boldsymbol{Z}}_{11}^{-1} \boldsymbol{b}_{1}
$$

and we get

$$
\boldsymbol{X}_{1}=\left[\overline{\boldsymbol{I}}-\left(\sum_{q=0}^{Q} \overline{\boldsymbol{M}}_{c, 21}^{q}+\sum_{q=0}^{Q} \overline{\boldsymbol{M}}_{c, 31}^{q}\right)\right]^{-1} \overline{\boldsymbol{Z}}_{11}^{-1} \boldsymbol{b}_{1},
$$

where the characteristic matrices are given by

$$
\begin{aligned}
\sum_{q=0}^{Q} \overline{\boldsymbol{M}}_{c, 21}^{q}= & \overline{\boldsymbol{Z}}_{11}^{-1} \overline{\boldsymbol{Z}}_{21}\left(\sum_{q=0}^{Q} \overline{\boldsymbol{M}}_{c, 32}^{q}\right) \times \\
& \overline{\boldsymbol{Z}}_{22}^{-1}\left(\overline{\boldsymbol{Z}}_{12}-\overline{\boldsymbol{Z}}_{12}^{\prime}\right)
\end{aligned}
$$

and

$$
\begin{aligned}
& \sum_{q=0}^{Q} \overline{\boldsymbol{M}}_{c, 31}^{q}=\overline{\boldsymbol{Z}}_{11}^{-1} \overline{\boldsymbol{Z}}_{31} \overline{\boldsymbol{Z}}_{33}^{-1} \times \\
& {\left[\overline{\boldsymbol{Z}}_{13}-\overline{\boldsymbol{Z}}_{23}\left(\sum_{q=0}^{Q} \overline{\boldsymbol{M}}_{c, 32}^{q}\right) \overline{\boldsymbol{Z}}_{22}^{-1}\left(\overline{\boldsymbol{Z}}_{12}-\overline{\boldsymbol{Z}}_{12}^{\prime}\right)\right]}
\end{aligned}
$$

Finally,

$$
\boldsymbol{X}_{1}=\sum_{p=0}^{P}\left(\sum_{q=0}^{Q} \overline{\boldsymbol{M}}_{c, 21}^{q}+\sum_{q=0}^{Q} \overline{\boldsymbol{M}}_{c, 31}^{q}\right)^{p} \overline{\boldsymbol{Z}}_{11}^{-1} \boldsymbol{b}_{1},
$$

Note that the obtained results are exactly the same as those of the GPILE method presented in this paper.

(ii) We first take $\boldsymbol{X}_{2}$ in the second equation, which we inject into the third equation. This allows us to express $\boldsymbol{X}_{3}$ as a function of $\boldsymbol{X}_{1}$. The expression of $\boldsymbol{X}_{3}$ is then introduced in the second equation and gives explicitly $\boldsymbol{X}_{2}$ as a function of $\boldsymbol{X}_{1}$. The expressions of $\boldsymbol{X}_{2}$ and $\boldsymbol{X}_{3}$ introduced in the first equation give the relation (41) where the characteristic matrices become

$$
\begin{aligned}
& \sum_{q=0}^{Q} \overline{\boldsymbol{M}}_{c, 21}^{q}=\overline{\boldsymbol{Z}}_{11}^{-1} \overline{\boldsymbol{Z}}_{21} \overline{\boldsymbol{Z}}_{22}^{-1} \times \\
& {\left[\overline{\boldsymbol{Z}}_{12}-\overline{\boldsymbol{Z}}_{32}\left(\sum_{q=0}^{Q} \overline{\boldsymbol{M}}_{c, 23}^{q}\right) \overline{\boldsymbol{Z}}_{33}^{-1}\left(\overline{\boldsymbol{Z}}_{13}-\overline{\boldsymbol{Z}}_{13}^{\prime}\right)\right]}
\end{aligned}
$$

and

$$
\begin{aligned}
\sum_{q=0}^{Q} \overline{\boldsymbol{M}}_{c, 31}^{q}= & \overline{\boldsymbol{Z}}_{11}^{-1} \overline{\boldsymbol{Z}}_{31}\left(\sum_{q=0}^{Q} \overline{\boldsymbol{M}}_{c, 23}^{q}\right) \times \\
& \overline{\boldsymbol{Z}}_{33}^{-1}\left(\overline{\boldsymbol{Z}}_{13}-\overline{\boldsymbol{Z}}_{13}^{\prime}\right)
\end{aligned}
$$

with

$$
\overline{\boldsymbol{M}}_{c, 23}=\overline{\boldsymbol{Z}}_{33}^{-1} \overline{\boldsymbol{Z}}_{23} \overline{\boldsymbol{Z}}_{22}^{-1} \overline{\boldsymbol{Z}}_{32} .
$$

Then, we deduce the surface currents on the object and the lower interface

$$
\begin{aligned}
\boldsymbol{X}_{2} & =\overline{\boldsymbol{Z}}_{22}^{-1} \overline{\boldsymbol{Z}}_{32}\left(\sum_{q=0}^{Q} \overline{\boldsymbol{M}}_{c, 23}^{q}\right) \overline{\boldsymbol{Z}}_{33}^{-1}\left(\overline{\boldsymbol{Z}}_{13}-\overline{\boldsymbol{Z}}_{13}^{\prime}\right) \boldsymbol{X}_{1} \\
& -\overline{\boldsymbol{Z}}_{22}^{-1} \overline{\boldsymbol{Z}}_{12} \boldsymbol{X}_{1}
\end{aligned}
$$

and

$$
\boldsymbol{X}_{3}=-\left(\sum_{q=0}^{Q} \overline{\boldsymbol{M}}_{c, 23}^{q}\right) \overline{\boldsymbol{Z}}_{33}^{-1}\left(\overline{\boldsymbol{Z}}_{13}-\overline{\boldsymbol{Z}}_{13}^{\prime}\right) \boldsymbol{X}_{1} .
$$

The obtained results are also equivalent to the GPILE method presented in this paper.

To have expressions which show the symmetry of the problem, we choose to take the expressions (34) and (46) for the surface currents on the object and on the lower surface (therefore (39) and (43) for the characteristic matrices). Thus, we obtain expressions which have a physical interpretation conforming to the PILE, EPILE and GPILE methods.

\section{ACKNOWLEDGMENT}

The authors acknowledge the support of the French Agence Nationale de la Recherche (ANR) through the project ACIMP (Improvement of knowledge of complex media composed of cracks and multiparameter inversion) under reference ANR18-CE22-0020. 


\section{REFERENCES}

[1] J. Jin, The Finite Element Method in Electromagnetics. John Wiley and Sons, 1993

[2] J. L. Volakis, A. Chatterjee, and L. C. Kempel, Finite Element Method for Electromagnetics, IEEE Press, 1998.

[3] Kane S. Yee, Numerical solution of initial boundary value problems involving Maxwell equations in isotropic media. IEEE Trans. on Antennas and Propagation, 14 (3): pp. 302307, 1966.

[4] James B. Cole and Saswatee Banerjee, Computing the Flow of Light: Nonstandard FDTD Methodologies for Photonics Design SPIE PRESS BOOK, 2017.

[5] F. Harrington, Field Computation by Moment Methods, Macmillan, New York, 1968.

[6] C. Bourlier, N. Pinel, and G. Kubické, Method of Moments for 2-D Scattering Problems. Basic Concepts and Applications. Hoboken, NJ, USA: Wiley, 2013, ser. Focus Series in Waves.

[7] D. A. Kapp and G. S. Brown, A new numerical method for rough-surface scattering calculations, IEEE Trans. Antennas Propag. 44, pp. 711722, 1996.

[8] D. Holliday, L. L. DeRaad Jr., and G. J. St-Cyr, Forward-backward: a new method for computing low-grazing angle scattering, IEEE Trans. Antennas Propag. 44, pp. 1199-1206, 1995.

[9] A. Iodice, Forward-backward method for scattering from dielectric rough surfaces, IEEE Trans. Antennas Propag. 50, pp. 901-911, 2002.

[10] H. T. Chou and J. T. Johnson, A novel acceleration algorithm for the computation of scattering from rough surfaces with the forward-backward method, Radio Sci. 33, pp. 1277-1287, 1998.

[11] L. Tsang, C. H. Chang, and H. Sangani, A banded matrix iterative approach to Monte Carlo simulations of scattering of waves by large scale random rough surface problems: TM case, Electron. Lett. vol 29, pp. 1666-1667, 1993.

[12] L. Tsang, C. H. Chang, H. Sangani, A. Ishimaru, and P. Phu, A banded matrix iterative approach to Monte Carlo simulations of large scale random rough surface scattering: TE case, J. Electromagn. Waves Appl. vol 29, pp. 1185-1200, 1993.

[13] L. Tsang, C. H. Chan, K. Pak, and H. Sangani, Monte-Carlo simulations of large-scale problems of random rough surface scattering and applications to grazing incidence with the BMIA/canonical grid method, IEEE Trans. Antennas Propag., vol. 43, pp. 851-859, 1995.

[14] C.-H. Kuo and M. Moghaddam, Scattering from multilayer rough surfaces based on the extended boundary condition method and truncated singular value decomposition, IEEE Trans. Antennas Propag., vol. 54, No. 10, pp. 29172929, 2006

[15] C. D. Moss, T. M. Grzegorczyk, H. C. Han, and J. A. Kong, Forward backward method with spectral acceleration for scattering from layered rough surfaces, IEEE Trans. Antennas Propag., vol. 54, no. 10, pp. 29172929, 2006.

[16] M. El-Shenawee, Polarimetric scattering from two-layered twodimensional random rough surfaces with and without buried objects, IEEE Trans. Geosci. Remote Sens., vol. 42, No. 1, pp. 67-76, 2004.

[17] N. Déchamps, N. De Beaucoudrey, C. Bourlier, and S. Toutain, Fast numerical method for electromagnetic scattering by rough layered interfaces: Propagation-inside-layer expansion method, J. Opt. Soc. Am. A vol. 23, no. 2, pp. 359-369, 2006.

[18] N. Déchamps and C. Bourlier, Electromagnetic scattering from a rough layer: Propagation-inside-layer expansion method combined to an updated BMIA/CAG approach, IEEE Trans. Antennas Propag., vol. 55, pp. $27902802,2007$.

[19] N. Déchamps and C. Bourlier, Electromagnetic scattering from a rough layer: propagation-inside-layer expansion method combined to the forward-backward novel spectral acceleration, IEEE Trans. Antennas Propag. vol 55, pp. 3576-3586, 2007.

[20] C. Bourlier, G. Kubické, and N. Déchamps, A fast method to compute scattering by a buried object under a randomly rough surface: PILE combined to FB-SA, J. Opt. Soc. Am. A 25, pp. 891-902, 2008.

[21] G. Kubické, C. Bourlier, and J. Saillard, Scattering by an object above a randomly rough surface from a fast numerical method: extended PILE method combined to FB-SA, IEEE Transactions on Antennas and Propagation, vol. 18, No. 03, pp. 495-519, 2008.

[22] C. Bourlier, C. Le Bastard, and V. Baltazart, Generalization of PILE Method to the EM Scattering From Stratified Subsurface With Rough Interlayers: Application to the Detection of Debondings Within Pavement Structure, IEEE Trans. Geoscience, and remote sensing, vol 53, No. 7, July, 2015.
[23] E. I. Thorsos, The validity of the Kirchhoff approximation for rough surface scattering using a Gaussian roughness spectrum, J. Acoust. Soc. Am. 83, pp. 78-92, 1988.

[24] C. Bourlier, C. Le Bastard, and N. Pinel, Full wave PILE method for the electromagnetic scattering from random rough layer, Proc. GPR Int. Conf. Ground Penetrating Radar, pp. 545-551, 2014.

Marc Songolo was born in Lubumbashi, Democratic Republic of the Congo (DRC), in 1984. He received the Graduate degree (2007) and Licence degree (2009) in Mathematics from the University of Lubumbashi. He received the Master degree (2014) in Applied Mathematics from the University of Kinshasa (DRC). He is a Researcher at Icam Ouest School of Engineering, working in Electrical Engineering Team. He is currently doing his $\mathrm{PhD}$ at IETR Laboratory, Polytech Nantes (University of Nantes), Nantes, France. His research focuses on numerical methods for the electromagnetic scattering by objects buried between random rough surfaces

Nicolas Pinel was born in Saint-Brieuc, France, in 1980. He received the Engineering degree and the M.S. degree in electronics and electrical engineering both from Polytech Nantes (University of Nantes), Nantes, France, in 2003 and the Ph.D. degree from the University of Nantes, in 2006. He is currently working as a Researcher at Icam Ouest School of Engineering, Nantes. His research interests are in the areas of microwave and optical remote sensing, propagation. In particular, he works on asymptotic methods of electromagnetic wave scattering from rough surfaces and layers, and its application to oil slicks on sea surfaces at moderate and grazing incidence angles.

Christophe Bourlier was born in La Fléche, France, in 1971. He received the M.S. degree in electronics from the University of Rennes, Rennes, France, in 1995 and the Ph.D. degree from the SEI Laboratory, Nantes, France, in 1999. He is currently with the IETR Laboratory, Polytech Nantes (University of Nantes), Nantes, France. He is a Researcher with the National Center for Scientific Research, working on electromagnetic wave scattering from rough surfaces (ocean-like surfaces) and objects for microwaves and infrared remote sensing applications and radar signatures. He is the author of more than 260 journal articles and conference papers. 